\title{
Interactive comment on "Multiphase
}

\section{MCM/CAPRAMmodeling of formation and}

\section{processing of secondary aerosol constituents}

\section{observed at the Mt. Tai summer campaign 2014"} by Yanhong Zhu et al.

Yanhong Zhu et al.

herrmann@tropos.de

Received and published: 28 February 2020

The comment was uploaded in the form of a supplement:

https://www.atmos-chem-phys-discuss.net/acp-2019-982/acp-2019-982-AC1-

supplement.pdf 